englischen Autoren dargestellt wird, erörtert hat, fügt er am Schlusse seiner Abhandlung einen kleinen Bericht über eigene Versuche am $\mathrm{Kaninchen} \mathrm{bei,} \mathrm{in} \mathrm{denen} \mathrm{er} \mathrm{auffallenderweise} \mathrm{nicht} \mathrm{zu} \mathrm{den-}$ selben Resultaten wie seine Vorgänger, besonders Fraser, gekommen ist. Wiewohl nun Langgaard selber, trotz der Differenzen beim Thierversuch, die Ergebnisse Fraser's, besonders sofern sie aus den Erfahrungen am Krankenbett herrühren, nicht im geringsten anzweifelt, so halten wir es doch mit Rücksicht auf einzelne eigene Resultate für angezeigt, die Hauptsache kurz hier zu reproduciren:

Bei subcutaner Verwendung (von Tinctura Srophanthi) blieben kleine Dosen vollkommen wirkungslos auf den Blutdruck; grössere Dosen führten entweder ein continuirliches Sinken des Blutdruckes bis zum Ende herbei, oder es kan zu einer kurz

III. Aus dem städtischen allgem. Krankenhause Friedrichshain, Abtheilung des Herrn Professor Fürbringer. \section{Zur Würdigung des therapeutischen Werthes der Strophanthustinctur.}

\section{Von Dr. H. Hochhaus, Assistenzarzt.}

Die grosse Zahl der Arzneimittel, welche währeud weniger Jahre als wirksamer Ersatz fïr die Digitalis empfohlen worden, ist letzthin durch den bekannten Pharmakologeu Fraser in Edinburg um eil neues vermehrt worden; dasselbe wird gewonnen aus dem Samen einer Strauchart - Strophanthus Kombé - die hauptsächlich in Ost-Afrika heimisch ist. Das wirksame Princip wurde von Fraser isolirt und als ein Glykosid von schwachsaurer Reaction erkannt, leicht löslich in Wasser und Alkohol, unlöslich in Aether und Chloroform. Am Hermuskel zeigten mit diesem Stro $\mathrm{ph}$ an th in angestellte Versuche: Bei kleinen Dosen Verstärkung der Systole, Verlangsamung der Herzaction und Steigerung des Blutdrucks; bei grösseren Dosen Stillstand in Systole und völlige Lähmung des Herzmuskels; keine Wirkung iibt Strophanthin, entgegen der Digitalis, auf das Gefässsystem aus; vergleichende Experimente zwischen Strophanthin und Digitalis erwiesen, dass ersteres in weit geringerer Dosis $(1: 6000000)$ Herzstillstand herbeiführt, als letzteres $(1: 4000$ noch nicht) dasselbe mithin ein weit stärkeres Herzgift ist.

Die Erfahrungen, welche. der vorgenannte Autor bei Herzerkrankungen der verschiedensten Art mit dem neuen Heilmittel machte, waren ausserordentlich günstige; fast durchweg gelang es $\mathrm{ihm}$, eine Verlangsamung der Herzaction, eine ausgiebigere systolische Contraction, damit Steigerung des Blutdruckes und Vermehrung der Diurese zu erzielen. Der Erfolg trat schnell und prompt ein, hielt längere Zeit an, und zeigte das Mittel auch bei ausgedehntem, lang fortgesetztem Gebrauch keine ungünstigeu Nebenwirkungen. Die Art und Weise der Anwendung war zum geringen Theil Injection in das Unterhautzellgewebe, weil dieselbe sehr schmerzhaft ist und starke Reizerscheinungen in der Haut verursacht; meist bediente sich Fraser einer alkoholischen Tinctur, anfangs im Verhältuiss $1: 8$, später $1: 20$; letztere empfiehlt er als die brauchbarste.

Auf diese Empfehlungen hin wurde das neue Mittel von einer Anzahl englischer und amerikanischer Aerzte angewendet und einstimmig dahin begutachtet, dass es bei incompensirten Klappenfehlern, ferner bei Erkrankungen des Herzmuskels ohue Klappenfehler vorzüglich geeignet sei, die geschwächte Herzaction zu stärken und zu reguliren, den Blutdruck zu steigern und die Diurese in Gang zu bringen; störende Nebenerscheinungen traten nie hervor, so dass es der Digitalis wenigstens gleichzustehen, sogar wegen letzterer Eigenschaft vorzuziehen schien.

In Deutschland berichtete über die neue Drogne zuerst, besonders, was die botanischen und pharmakologischen Eigenschaften angeht, in ausführlicher Weise Langgaard, ${ }^{3}$ ) dem wir auch die kurzen vorstehenden Notizen grösstentheils entnommen haben; nachdem derselbe die Wirkung des Strophanthussaamen, wie sie von

1) Prof. E. Wagner theilte in der Discussion 2 einschlägige Fäle mit, bei denen die Intussusception vom Rectum her durch äussere Manipulationen beseitigt werden konnte und Heilung erfolgte.

${ }^{2}$ ) In No. 30 des chirurg. Centralbl. plaidirt L. Rehn dafür, dass jeder Fall von Ileus alsbald chirurgischer Behandlung überlassen und mögJichst in den ersten 24 Stunden operirt werde. Auf Grund zweier Fälle hält er sich zu dieser Empfehlung berechtigt, da erst nach voransoeschicktem Bauchschnitt die Magen-Ausspullung recht zur Geltung komme, weil der atmosphärische Druck jetzt stärker cinwirken könne. Mir scheint, dass diese Erklärung entsehiedene $Z$ weifel zulässt; nach meiner Ansicht kann die Spannung der Bauchmuskeln - bezw. die Banchpresse die Wirkung der Magen-Ausspülung fördern und der atmosphärische Druck nach Relaxation der Banchmuskeln in der Narkose mindestens ebenso kräftig sich äusseru, als nach Eröffung der Bauchhohle. Ich halte es aber für gewagt, auf 2 derartige Beobachtungen hin, jene Massnahmen fül alle Fälle von Ilens zu empfehlen.

3) Therapeutische Monatshefte No. 5, 1887. vorübergehenden, mässigen Steigerung. Eine 'erhebliche Erhöhung des Blutdruckes sah ich meistens erst nach einer Periode bedeutender Druckschwankungen und unregelmässiger Herzaction kurz vor dem durch Herzlällmung erfolgenden Tode. Nur in einem Falle gelangten nach einer nicht tödlichen Gabe deutliche Vaguspulse und eine Drucksteigerung von 94 auf $104 \mathrm{~mm}$ Quecksilber zur Beobachtung. Bei direkter Infusion ganz kleiner Dosen kam es stets zu einer primären, nicht unerheblichen Blutdrucksenkung, und der weitere Verlauf gestaltete sich wie nach subcutaner Injection grösserer Dosen."

In einer späteren Mittheilung berichtet Langgaard ${ }^{1}$ ) über weitere Versuche mit Strophanthustinctur, die el mit Bahadhurji bei Kaninchen und Fröschen angestellt, um die Frage zu erledigen, ob das Mittel eine Wirkung auf das Nervensystem ausübt oder nicht, was bekanntlich von anderen Autoren (Drasche, Boyd) in letzterem Sinne beantwortet war. Auch hier konnte er im Widerspruch mit den oben genannten Autoren feststellen dass nach Einverleibung kleiner Dosen die betreffenden Thiere müde und schläfrig wurden, nach grösseren Dosen aber allgemeine Lähmungserscheinungen darboten; mithin an einer direkten Beeinflussung des Centralnervensystems kaum ein Zweifel blieb.

Weitere Erfahrungen über das neue Mittel am Krankenbett wurden zuerst von Drasche $\mathrm{C}^{2}$ ) in Wien, später von Pins ${ }^{3}$ ) gesammelt und veröffentlicht. Letzterer fasst seine Anschaunng, die er auf 12 Fälle von Erkrankungen des Herzens, des Gefässsystems und der Nieren basirt, in folgendes Resumé zusammen: In sämmtlichen Fällen war der Einfluss der Tinctur nnverkennbar, der Puls wurde voller und kräftiger, die Arhythmie machte einem gleichmässigen, ruhigen Pulse Platz. Die Frequenz des Pulses wurde um 12-40 Schläge in der Minute herabgesetzt, die Athembeklemmungen liessen nach, die Harnabsonderung wurde vermehrt, die Oedeme schwanden in wenig Tagen." Noch sicherer wie auf Puls und Diurese fand Pins die Wirkung gegen Dyspnoe und Asthma, wenn sie durch Erkrankungen des Herzens oder der Nieren bedingt waren stets linderte die Tinctur in kürzester Zeit die Beschwerden, und nach einem ein- bis zweiwöchentlichen Gebrauch kehrten die Anfälle während der Beobachtungsdauer nicht wieder. Die Wirkung war so prompt und zuverlässig, dass P. nicht ansteht, den Strophanthussaamen gegen diese Art der Dyspnoe als ein Specificum $z u$ empfehlen. Gewöhnung an das Mittel, cumulative Wirkung oder sonstige störende Nebenerscheinungen konnte P. nicht constatiren.

Weniger Wirkung zeigte sich in der Anwendung bei Ascites und Anasarca in Folge von Lebercirrhose, chronischer Peritonitis, und anderen Unterleibskrankheiten; desgleichen bei Herzklopfen und Pulsbeschleunigung auf nervöser Basis; auch bei acuter Pneumonie und bei Phthisis pulmonum war der Erfolg wenig hervortretend, wiewohl bei letzterer Krankheit eine Besserung des Appetits unverkennbar war. In Erwägung aller dieser Umstände kommt also Pins zu dem Endschluss, dass Strophanthus als herzkräftigendes und regulirendes Mittel bei Erkrankung des Herzmuskels, mit und ohne Klappenfehler, ferner bei Morb. Brightii der Digitalis mindestens gleichzustellen, wegen des Mangels jeder Nebenwirkung jedoch vorzuziehen sei; nur dort, wo es vorzugsweise auf eine diuretische Wirkung ankommt, giebt er der Digitalis den Vor"zug.

Nach diesen ermuthigenden Erfolgen lag eine Prüfung des Mittels nahe, trotz der vielen Enttäuschungen, die die meisten Ersatzmittel der Digitalis gebracht hatten.

Ein brauchbares Urtheil aber über den therapeutischen Werth oder Unwerth eines Mittels, welches nicht als Specificum wirkt, sondern die Thätigkeit eines kranken Organes resp. Organsystemes il bestimmter Richtung beeinflusst, kann nur an einer aus-

1) Therapeutische Monatshefte No. 8, 1887.

2) Wiener medicinische Blätter, 5. Mai 1887 und die folgenden Numerm.

3) Therapeutische Monatshefte No. 6, 1887. 
reichenden klinischen Beobachtung reifen; die unbefangene, praktische Erfahrung am Krankenbett entscheidet dann allein, sollte sie auch aller Theorie schnurstracks zuwider sein. In dieser Erwägung hat Herr Prof. Fürbringer die Nachprüfung über die Wirkung der Strophanthustinctur an einem ausgiebigeren Krankenmaterial, als die bisherigen Berichterstatter vorgeführt, auf der inneren Abtheilung des Allgemeinen Krankeuhauses veranlasst; - die Fälle sind mir gütigst zur Publication überlassen worden, und verfehle ich nicht, meinem verehrten Chef an dieser Stelle meinen besten Dank auszusprechen.

Wir legen hiermit die Resultate in ihren wichtigeren Grundzügen nieder, wie sie an einer grösseren Anzahl von Kranken ohne besondere Auswahl, die ausschliesslich von solchen Affectionen betroffen waren, bei denen das Herz, das Gefässsystem oder die Nieren vorzugsweise geschädigt waren, gewonnen wurden.

Um die Einwirkung des Mittels auch auf den gesunden Orgauismus festzustellen, hatten wir ausserdem noch an einigen Gesunden Versuche angestellt.

Im Ganzen bestand das Beobachtungsmaterial aus

I. 5 Gesunden,

II. 10 incompensirten Herzklappenfehlern,

III. 18 chronischen Degenerationen des Herzmuskels ohne Klappenerkrankung,

IV. 19 Nierenerkrankungen,

V. 8 anderen Affectionen des Herzens, die nicht unter die ebengenannten Kategorieen fallen,

im Ganzen also aus 60 Fällen.

Das Präparat, dessen wir uns bedienten, war ausschliesslich die alkoholische Tinctur, im Verhältniss von $1: 20$, durch die hiesigen Firmen Brückner \& Lampe, Riedel von Merk in Darmstadt bezogen.

Bei den 5 Gesunden war in 2 Fällen gar keine Wirkıng zu constatiren, weder auf Puls noch auf Diurese; bei den 3 übrigen war eine vorübergehende Steigerung der Urinmenge, sowie bei 2 auch eine deutliche Pulsverlangsamung ausgesprochen; eine Beeinflussuug anderer Organe war nicht nachweisbar.

Beispiel: Frau E., 34 Jahre alt, kräftig, wohlgenährt, sämmtliche Organe normal; wegen eines leichten Muskelrheumatismus dem Krankenhause zugewiesen. Während des Versuchs, dessen Anordnung und Resultat aus nachfolgender Tabelle ersichtlich, bekam sie stets dieselbe Diät und musste völlige Bettruhe innehalten.

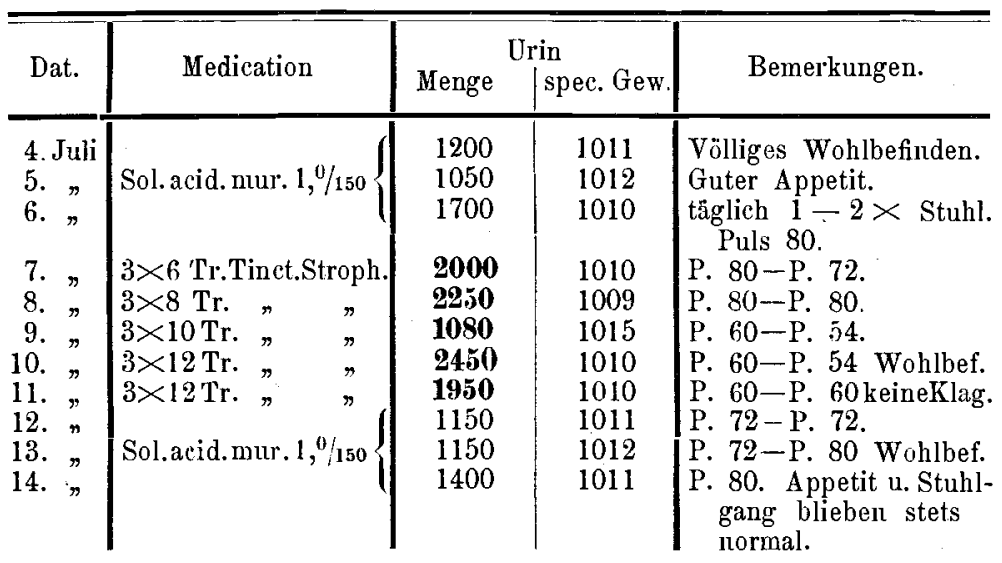

Unter den 10 Klappenfehlern, sämmtlich im Stadium der Compeusationsstörung, mit frequenter, irregulärer Herzaction, weichem, leicht uuterdrückbarem Pulse, mit starker Dyspnoe und Oedemen, war ein sehr guter Erfolg $u$ u in 2 Fällen zu constatiren, insofern die Kranken nach kurzer Behandlung mit Strophanthus sich in jeder Beziehung derartig gekräftigt fühlten, dass sie bald aus dem Krankenhause als wesentlich gebessert entlassen werden konnten. Iu 2 Fällen war der Erfolg zwar auch deutlich, der Puls wurde langsam und kräftig, die Dyspnoe besserte sich, aber die Oedeme schwanden nicht vollkommen, so dass die Pat. trotz längerer Behandlung allemal beim Aufstehen und Herumgeheı wieder Oedeme bekamen und dyspnoisch wurden. In 5 Fällen war eine Wirkung der Strophanthustinctur überhaupt nicht zu constatiren.

Bei einer Kranken war das Medicament von ausgezeichneter Wirkung sowohl auf Herz, als Diurese und Athmung; Pat. konnte bereits aufstehen, starb dann aber ganz plötzlich, ohne dass die Section irgend einen Aufschluss über den plötzlichen Exitus letalis ergeben hätte.

'Zur näheren Beleuchtung der Strophanthuswirkung lassen wir einige Krankengeschichten im Auszuge folgen:

Francisca G., 30 J., leidet seit vieleı Jahren häufig an Herzklopfen, kurzer Luft und Anschwellung der Beine: jetzt ist sie bereits 2.1 Hause zwei Wochen dieserhalb bettlägerig und auch schon ärztlich behandelt, aber ohne Erfolg.

Stat. praes.: gracil gebaute, cyanotische Frau; an den unteren Extremitäten Oedeme; starke Dyspnoe, trockener Husten. Am Herzen die Zeichen einer Mitralinsufficienz. Puls weich, frequent, arhythmisch.

\begin{tabular}{|c|c|c|c|c|}
\hline \multirow{2}{*}{ Dat. } & \multirow{2}{*}{ Medication } & \multicolumn{2}{|c|}{ Urin } & \multirow{2}{*}{ Bemerkungen. } \\
\hline & & Menge & Gew. & \\
\hline 18. Aug & $3 \times 8$ Tr.Tinct. Stroph & 300 & 1020 & \\
\hline 19. & $3 \times 10^{\prime}$ Tr. & 1000 & 1012 & \\
\hline 20. & $3 \times 12$ Tr. & 1900 & 1010 & $\begin{array}{l}\text { Puls krätiger,langsamer } \\
\text { aber noch arhythmiseh } \\
\text { Dy sp no e gering. }\end{array}$ \\
\hline 21, & $3 \times 12$ Tr. & $\mathbf{2 8 5 0}$ & 1007 & Besserung sehreitet fort \\
\hline 22, & & 2500 & 1006 & \\
\hline 23. & & 2400 & 1006 & $\begin{array}{l}\text { Puls langsam, voll, } \\
\text { kräftig, und rege el- } \\
\text { mässig. }\end{array}$ \\
\hline & Roborantia & 2600 & 1007 & $\begin{array}{l}\text { Oedeme verschwunden } \\
\text { lieine Dyspnoe. }\end{array}$ \\
\hline 25. & & 1200 & 1010 & Das Wohlbef. hält an \\
\hline $\begin{array}{l}26 . " \\
27 . "\end{array}$ & & $\begin{array}{l}1020 \\
2050\end{array}$ & 1007 & $\begin{array}{l}\text { Der Stuhl war stets nor- } \\
\text { mal, ohne Urinverlust, } \\
\text { Appetit gut. }\end{array}$ \\
\hline 28 & & 1500 & 1011 & \\
\hline
\end{tabular}

Der Einfluss auf die Diurese trat am 2. Tage der Medication ein, am 3. Tage war die Besserung des Pulses und der Respiration ausgesprochen, während dagegen der Puls erst am 6. Tage regelmässig wurde, ein Verhalten, das uns überhaupt mehrmals auffiel, der Einfluss auf die Frequenz der Herzthätigkeit war viel früher ausgesprochen, als der auf den Rhythmus; die Athrnung wurde am 3. Tage ruhig. Die Besserung war anhaltend, so dass Pat. bald wesentlich gebessert entlassen werden konnte.

Frau D., 44 Jahre, leidet seit längerer Zeit an starker Athemnoth, Herzklopfen und geschwollenen Beinen; schon mit Digitalis und Coffein behandelt, aber mit nur temporärem Erfolg.

Stat. präs.: Krăftige, adipöse Frau; an den unteren Extremitäten Oedeme, heftige Dyspnoe.

Herzbefund: Insufficientia mitral, incompensata.

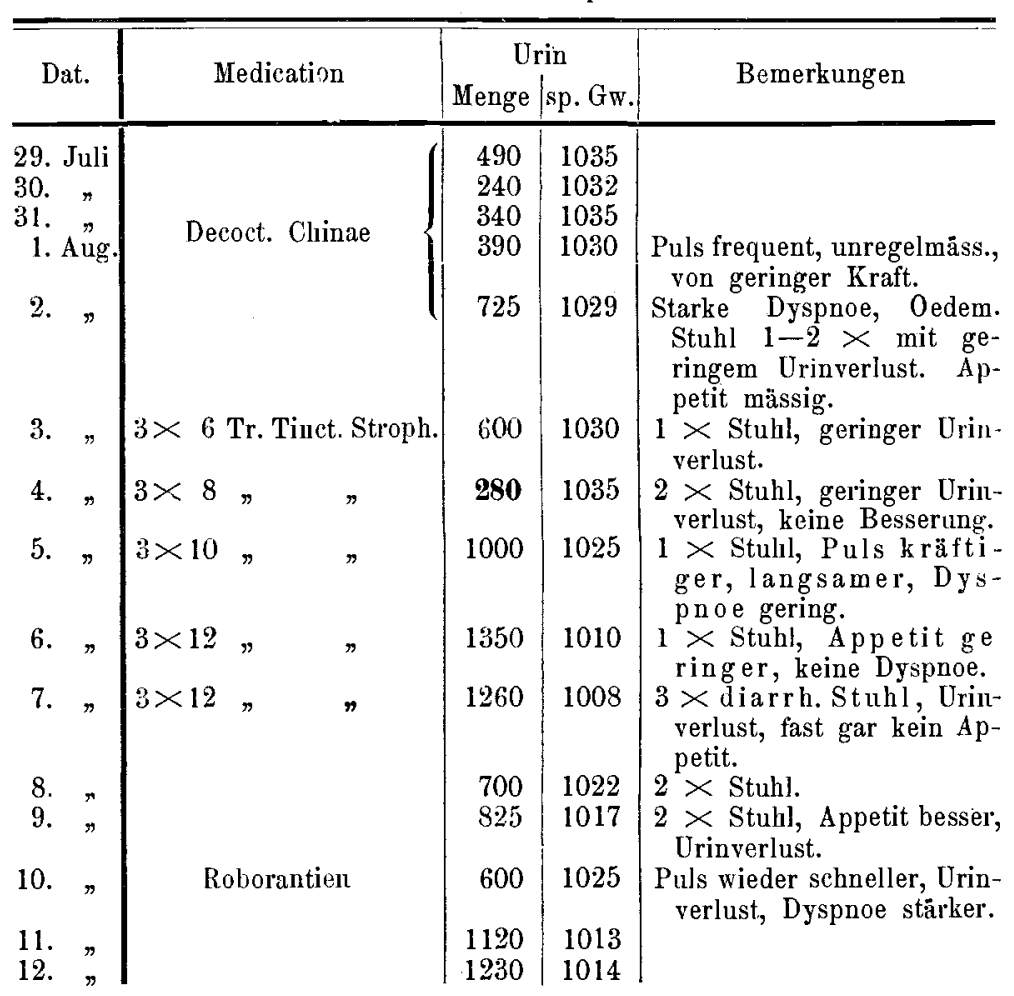

Es trat die Wirkung am 3. Tage hervor auf sämmtliche Krankheitserscheinungen, schwand aber auch bald wieder nach Aussetzen des Mittels; auffallend ist das am 2. Tage beobachtete Sinkell der Harnmenge, ein Factum, dem wir nicltt selten begegnet sind, und das uns mit den oben erwähnten Experimenten Langgaard's, in denen auch häufig direkt nach der Einverleibung der Blutdruck sank, wohl im Einklang zu stehen scheint. In den letzten Tagen klagt Pat. über Druck in der Magengegend und Appetitlosigkeit; der Stuhl wurde diarrhoisch.

Der Erfolg war also zwar deutlich, aber vorübergehend, später wurde Patient. durch Digitalis mit besserem und dauerndem Erfolg behandelt.

Frau T., 37 Jahre alt, schon mehrmals im hiesigen Irankenhause an Herzfehler behandelt, kommt wieder hinein wegen starker Athemnoth, Herzklopfen und Wassersucht.

Status präs.: Kräftig gebaute Frau, ödematös. 
Herzbefind: Insufficientia et Stenos. mitralis. Puls klein, frequent, arhythmisch, spärliche Diurese.

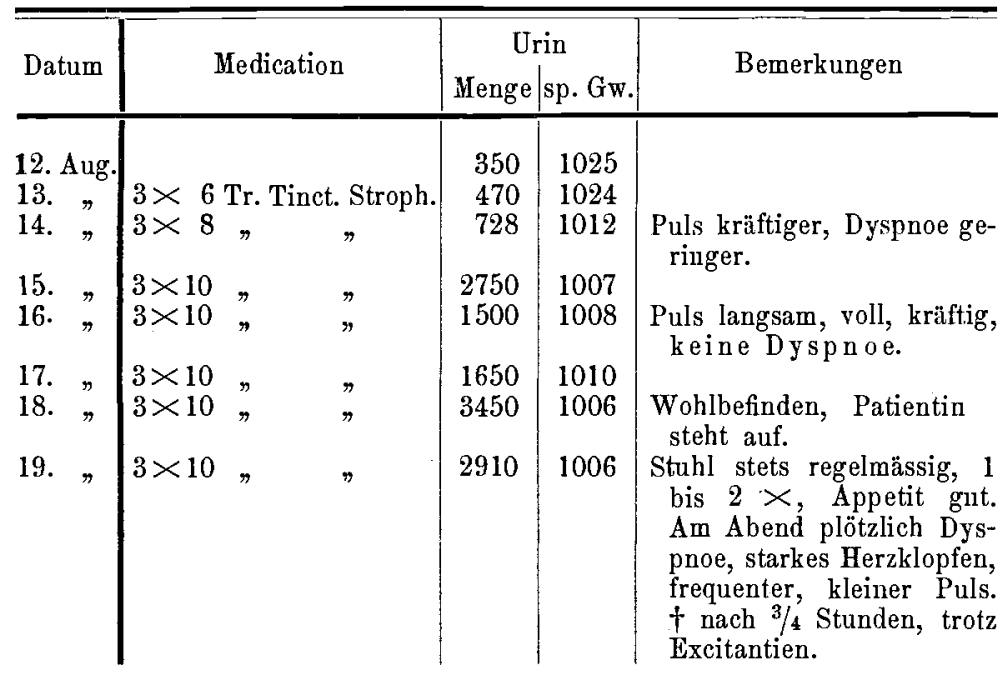

Strophanthus war, wie oben ersichtlich, von ausgezeichneter Wirkung. Die Patientin schien sehr wohl, stand bereits auf, bis der Tod plötzlich am 19. Abends eintrat. Die Section ergab: Stenosis et Insuffic. mitralis. Myocarditis chronica. Staungssorgane. Ob vielleicht die längere Zeit fortgebrauchte Dosis auf das Herz zuletzt nicht doch lähmend eingewirkt, wäre in diesem Falle wohl zu erwägen, zumal wir ja aus dem Thierversuch wissen, dass Strophanthus ein schon in minimaler Verdünnnng wirkendes Herzgift ist.

In 5 Fällen war, wie schon oben erwähnt, das Medicament völlig wirkungslos; in einem von diesen zeigte es sehr unangenehme Nebenwirkungen.

Der Kranke, welcher bis dahin stets leidlichen Appetit gehabt, klagt am 2. Tage über Aufstossen und Appetitlosigkeit, der Stuhl wird diarrhoisch; am 3. Tage war der Appetit völlig geschwunden, Patient brach einmal und hatte 4 diarrhoische Stühle, so dass das Mittel ausgesetzt werden musste.

Im Ganzen ist die Wirkung der Strophanthustinctur bei incompensirten Klappenfehlern eine $\mathrm{mässig}$ zufriedenstellende; in den günstigen Fällen wurde der Puls bald langsamer und kräftiger, die Diurese kam in Gang, und die Oedeme verschwanden; störend waren die zuweilen auftretenden dyspeptischen Erscheinungen und die Diarrhoe.

Mehrere Kranke, bei denen das Mittel wirkungslos, wurden später erfolgreich mit Digitalis behandelt.

Die folgende Gruppe umfasst diejenigen Erkrankungen des Herzens, bei welchen ein Klappenfehler zwar nicht zu constatiren, bei denen die physikalische Untersuchung meist ergiebt: eine Verbreiterung der Herzdämpfung nach rechts und links in schwankender Ausdehnung; die Herztöne sind leise und geräuschartig; der Puls ist in der Regel weich, klein und arhythmisch; dabei ist über der Lunge häufig Bronchitis, unter Umständen mit emphysematischer Blähung ausgesprochen, die Athrnung ist frequent, die Diurese gering und es besteht Wassersucht, - also das Bild derjenigen Affection, die wir als Myocarditis chronica zu bezeichnell pflegen; von dieser haben wir 18 Fälle mit Tinctura Strophanthi behandelt.

Bei 8 von diesen war gar kein Erfolg ersichtlich, bei 2 wurde der Puls sogar deutlich schwächer und die Diurese verringert; in einem Falle waren die dyspeptischen Beschwerden (Appetitlosigkeit, Erbrechen), sowie die Diarrhoe derart heftig, dass das Mittel dieserhalb ausgesetzt werden musste; bei 2 Kranken konnte trotz leichter Dyspepsie die Medication, freilich erfolglos, fortgesetzt werden.

Bei den übrigen 10 Kranken war der Erfolg augenscheinlich, der Puls wurde in Kürze kräftiger, langsam und regelmässig; die Verlangsamung des Pulses betrug durchschnittlich 20-30 Schläge in der Minute, in einem Falle fiel der Puls sogar von 90 auf 44; die Diurese wurde in manchen Fällen erheblich gesteigert; noch mehr fiel auf die prompte Beseitigung der Dyspnoe durch das Mittel, häufig, noch ehe Puls und Diurese eine Besserung erkennen liessen, durfte der Kranke, dem bisher nur Morphium Ruhe verschafft hatte, sich eines ruhigen, durch keinen Dyspnoe-Anfall gestörten 'Schlafes erfreuen.

Der Erfolg war ziemlich lang anhaltend und nur bei $3 \mathrm{~Pa}$ tienten durch vorübergehende Verdauungsstörungen beeinträchtigt.

Einige Beispiele werden das vorhin Gesagte erläßtern:

Eduard B., 37 Jahre alt, kräftig gebaut, mit gut entwickeltēr Muskulatur. Herz nach rechts bedeutend vergrössert; Töne rein, aber leise. Puls sehr klein, frequent, unregelmässig. Cyanose, heftige Dyspnoe; Anasarca am ganzen Körper.

Bisher mit Digitalis bei gutem, aber temporärem Erfolg behandelt.

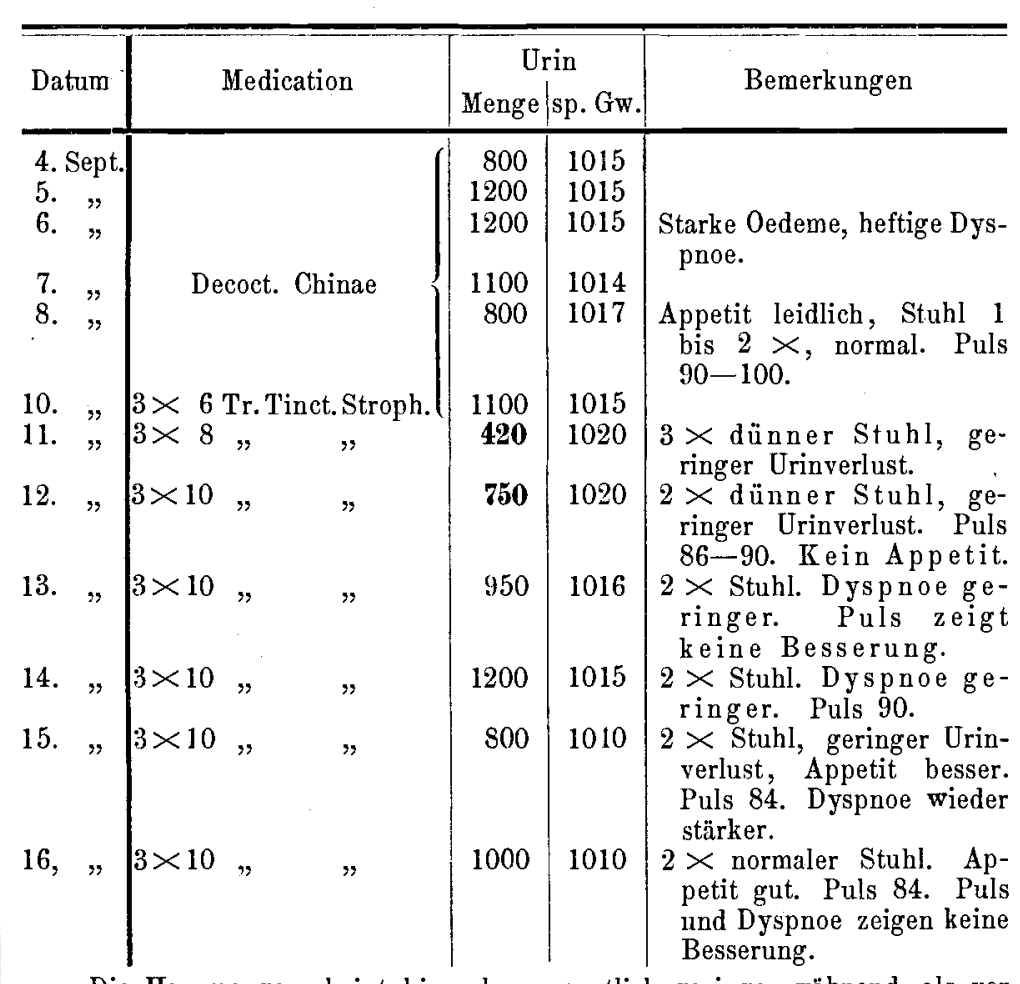

Die Harnmenge scheint hier also wesentlich geringer während, als vor der Strophanthus - Darreichung. Der Puls fiel um 6 Schläge; die Qualität besserte sich gar nicht, im Gegentheil schien derselbe eher etwas debiler als zuvor. Die Oedeme blieben, in den ersten Tagen traten anch hier Dyspepsie und Diarrhoe auf, die aber trotz der Fortsetzung der Medication sich besserten. Besonders möchten wir noch hervorheben, dass trotz der fehlenden Wirkung auf Herz und Diurese die Dyspnoe am 4. und 5. Tage der Medication deutlich geringer wurde und Patient sich etwas erleichtert fühlte, - eine Wirkung, die uns nach den vorerwähnten Versuchen Lang g a ard's über die Einwirkung der Drogne auf das Nervensystem wohl verständlich erscheint.

In noch höherem Maasse trat diese Erscheinung bei folgenden Kranken hervor:

Carl N., $67 \mathrm{Jahre}$ alt, stark ödematös am ganzen Körper, hat heftige Dyspuoe und ausgesprochene Cyanose.

Die physikalische Untersuchung ergiebt: Myocarditis chronica, kleiner frenquenter Puls. Hydrothorax beiderseits.

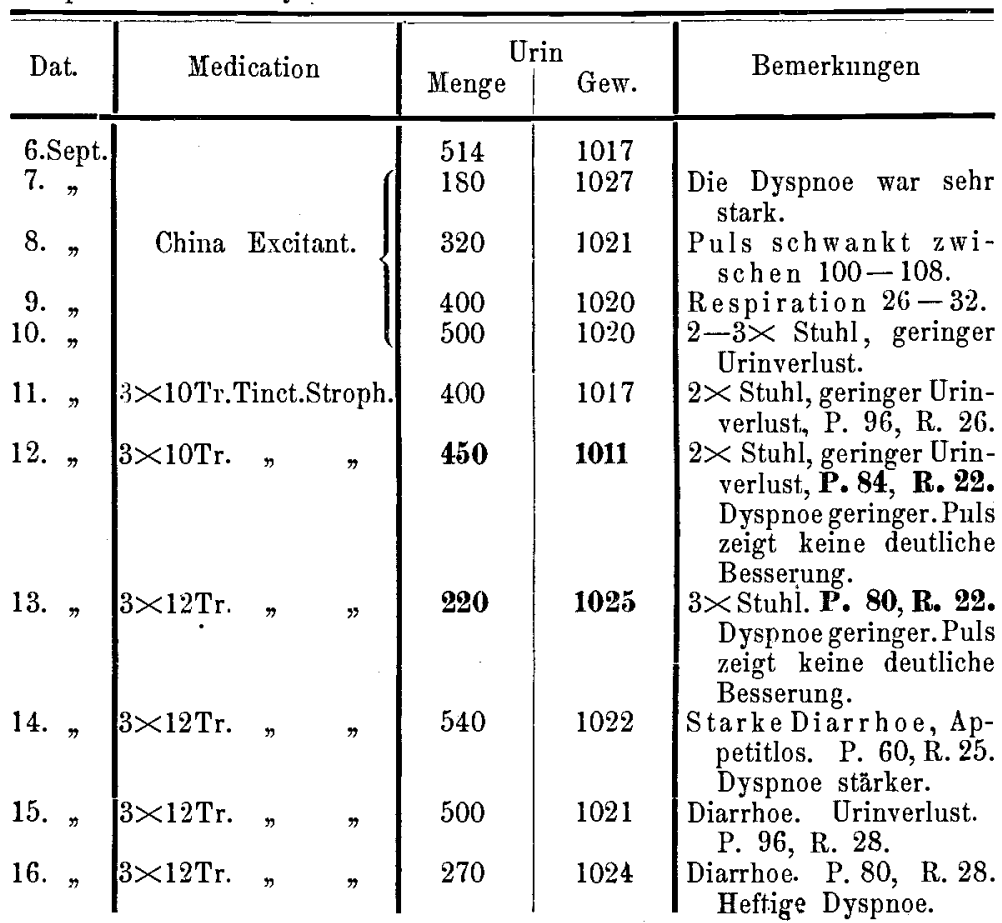

Die Verlangsamung des Pulses war ausgesprochen, doch die Kraft wenig besser, die Diurese nicht gesteigert, so dass wir ihm die Besserung der Athmung am 12. und 13. nicht zuschreiben können; wir sind im Gegentheil mit Langgaard der Meinung, dass dieser Umstand einer direkten Beeinflussung des Nervensystems zuzuschreiben ist; am 16. war der Puls auch nur 80 i. M., trotzdem war der Kranke wieder dyspnoisch, unserer Meinung deshalb, weil die Dosis jetzt zur hypnotischen Wirkung nicht mehr ausreichend war. Am 14. traten dann die schon öfters als störend erwähnten Nebenwirkungen der Diarrhoe ein.

(Schluss folgt.) 